\title{
Hypophosphatemia and Hyponatremia in Systemic Lupus Erythematosus Patients and Its Relation to Clinical Characteristic and Disease Activity
}

\author{
Alyaa A. Mohamed*, Esam M. Abu AlFadl, Osama S. Daifallah, Rabab H. Ali
}

Department of Physical Medicine, Rheumatology \& Rehabilitation, Faculty of Medicine, Sohag University, Egypt

*Corresponding author: Alyaa A. Mohamed, Mobile: (+20)1007218644, E-mail: alyaa.abdelhamed.m@gmail.com

\begin{abstract}
Background: Systemic Lupus Erythematosus (SLE) is a chronic auto-immune disease with diverse manifestations, ranging from mild rash or arthritis to severe organ-threatening involvement.

Objective: The aim of the work was to find-out the possible association of hypophosphatemia and hyponatremia with disease activity in SLE patients.

Patients and methods: A total of 100 patients with SLE were involved in this study and the serum level of sodium and phosphorus, erythrocyte sedimentation rate (ESR), leucocytic and platelet counts, and 24 hr. protein were measured. SLE disease activity index (SLEDAI) score was assessed,

Results: The majority of patients were females; 94 (94\%) and 6 males (6\%) (F:M 15.7:1). The age of the patient ranged from 17 to 63 years with a mean age of $34.23 \pm 11.19$ years. The disease duration was $48 \pm 55.7$ months. $7.41 \%$ of SLE patients were hyponatremic and $49 \%$ showed normonatremic. There was a significant correlation between Na level, SLEDAI score, vasculitis and arthritis and insignificant correlation with ESR. 47 patients were hypophosphatemic (47\%) and $53(53 \%)$ were normophophatemic. There was a significant correlation between phosphate level and SLEDAI, oral ulcers $\mathrm{p}=0.001$ and arthritis $\mathrm{p}<0.0004$ but negatively related with ESR.

Conclusion: It could be concluded that hyponatremia and hypophosphatemia are significantly related to SLEDAI, so it could be used as indicators of SLE activity and sever inflammation.

Keywords: Systemic lupus erythematosus, Hypophosphatemia, Hyponatremia, SLEDAI score.
\end{abstract}

\section{INTRODUCTION}

Systemic lupus erythematosus (SLE) is a chronic auto-immune disease with diverse manifestations, ranging from mild rash or arthritis to severe organ-threatening involvement ${ }^{(\mathbf{1})}$.

SLE in Egypt shares many similarities with that in other nations. At the same time, several different clinical and immunologic characteristics are displayed even within the same country. Autoantibodies are present in a comparable number of patients as shown worldwide. Genetic and/or climatic factors may lead to different presentations of lupus and subsequent different therapeutic regimens ${ }^{(2)}$.

Systemic lupus Erythematosus (SLE) is a typical autoimmune disease which adversely affects multiple end-organs, including heart, joints, liver, and kidneys. Hyper activation of autoantibodies against cell nucleic antigens leads to the deposition of immune complex in end organs ${ }^{(3,4)}$.

Common manifestations may include arthralgia and arthritis, malar and other skin rashes, pleuritis or pericarditis, renal or CNS involvement, hematologic cytopenia and weight changes are the most common symptoms in new cases or recurrent active SLE flares ${ }^{(5)}$, with abnormal laboratory data, such as pancytopenia, low serum complement concentrations and positive autoantibodies against nucleus and DNA. Abnormalities of phosphorus (P) have never been described ${ }^{(6,7)}$.
Lupus activity can be measured by many laboratory markers as aberrant production and imbalance of T-helper (Th1/Th2) cell cytokines which have been implicated in the pathogenesis of autoimmunity. Also Increased RDW is connected with active disease status of SLE patients. RDW could be used as a surrogate marker of the inflammation and correlated with the SLEDAI score ${ }^{(8)}$.

Hypophosphatemia is observed not only in genetic diseases such as $\mathrm{X}$-linked hypophosphatemia (9), autosomal dominant hypophosphatemia, and McCune-Albright syndrome ${ }^{(10)}$, but also in acquired states including tumor-induced osteomalacia and malnutrition. Studies have revealed that fibroblast growth factor 23 seems to play a key role in phosphate metabolism (11). Although mechanisms of hypophosphatemia in these conditions are not fully understood. A recent report showed that serum phosphate concentrations were decreased in early sepsis, and tumor necrosis factor $\alpha$ (TNF- $\alpha$ ) and interleukin 6 (IL-6) were suggested to be causes of hypophosphatemia in this pathologic condition ${ }^{(\mathbf{1 2})}$. In SLE, those cytokines have been shown to be involved in the disease activity, which led us to speculate about whether Phosphate metabolism in SLE might be aberrant, especially in patients with active disease ${ }^{\left({ }^{(9)}\right.}$.

Hyponatremia is defined as a serum sodium level that is less than $135 \mathrm{mEq} / \mathrm{L}$. It is a common water balance disorder, with multiple causes including, 
syndrome of inappropriate secretion of antidiuretic hormone (SIADH), diuretics use, adrenal insufficiency, liver cirrhosis and heart failure ${ }^{(13)}$. Hyponatremia is presented mainly by central nervous system (CNS) dysfunction and is more dramatic with acute and marked decrease of serum sodium (Na) levels. Hyponatremic encephalopathy occurs as the result of the brain swelling secondary to acute hyponatremia and is associated with $34 \%$ risk of mortality (14). Hyponatremia could reflect disease activity in children and adults with SLE ${ }^{(15)}$. Hyponatremia in SLE patients is related mainly to renal disease and the use of drugs as cyclophosphamide (CYC) ${ }^{(\mathbf{1 6})}$. However, hyponatremia in SLE has been reported and showed the association of SIADH with neuropsychiatric lupus ${ }^{(17,18)}$.

The aim of the present study was to find-out the possible association of hypophosphatemia and hyponatremia with disease activity in SLE patients.

\section{PATIENTS AND METHODS}

This study included a total of 100 adult SLE, attending at Department of Rheumatology, Sohag University Hospital.

Inclusion Criteria: Patients who fulfilled the criteria of Systemic Lupus International Collaborating Clinics (SLICC) classification ${ }^{(\mathbf{1 9})}$.

Exclusion Criteria: SLE patients with secondary antiphospholipid syndrome (APS) or with other autoimmune diseases. Also, Patients with renal, hepatic or heart failure and those taking CYC or diuretics were excluded.

\section{Ethical consent:}

An approval of the study was obtained from Sohag University academic and ethical committee. Every patient signed an informed written consent for acceptance of the operation. This work has been carried out in accordance with The Code of Ethics of the World Medical Association (Declaration of Helsinki) for studies involving humans.

Patients were divided according to serum Na levels into: Group I (hyponatremia $n=41$ ) with a level less than $135 \mathrm{mEq} / \mathrm{L}$ and those without hyponatremia $(\mathrm{n}=$ 59) group II. Also, the same patients were divided according to serum phosphorus levels into Group I (hypophosphatemia $\mathrm{n}=47$ ) with level of phosphorus less than 2.5 and those without (hypophosphatemia $n=53$ ). SLE disease activity index (SLEDAI) ${ }^{(20)}$ was assessed. Patients with a score less than 4 were considered in remission, while those with a score $>4$ were considered active $^{\text {(20). }}$. The number of active SLE patients were 83 while those in remission were 17 patients. Blood samples were drawn from all patients after they had fasted overnight. All laboratory analyses were performed on the same day within one hour of samples collection. Serum sodium, phosphorus, complements (C3 and C4) determination, complete blood count $(\mathrm{CBC})$, erythrocyte sedimentation rate (ESR), liver and kidney function tests were done. Serum, anti-nuclear antibodies (ANA) were quantitatively measured by immunofluorescence. Medications received by the patients were recorded. SLE disease activity index (SLEDAI) ${ }^{(20)}$ was assessed. A serum sodium level $<135$ $\mathrm{mEq} / \mathrm{L}$ was considered hyponatremia while a serum phosphorous level $<2.5 \mathrm{mEq} / \mathrm{L}$ was considered hypophosphatemia.

\section{Statistical analysis}

Data was computed and analyzed via IBMSPSS-20 computer program. Data was presented as means \pm standard deviation (SD), median and inter quartile range or numbers and percentages. The results were examined for normality via Shapiro-Wilk test. The Mann-Whitney test and Spearman's correlation were employed. Chi-square (v2) test and Fisher's exact test were considered. ROC/Regression. P-value $<0.05$ was considered significant.

\section{RESULT}

100 SLE patients were assessed by SLEDAI activity score and correlated with sodium were included in this study with 94 females (94\%) and 6 males (6\%) with a mean age of $34.2 \pm 11.19$ years. Patients with hyponatremia $(\mathrm{n}=41)$ were 5 males and 36females with a mean age of $33.2 \pm 9.67$ years while patients without (n $=59$ ) were 3 males and 56 females with a mean age of $34.84 \pm 12.3$ years. The clinical manifestations in patients with and without hyponatremia show insignificant correlation with ESR ( $\mathrm{p}=0.7)$, low platelet $(\mathrm{p}=0.5)$, low WBCs $(\mathrm{p}=0.09)$ and significant correlation with SELDIA score $(\mathrm{p}=<0.0001)$, vasculitis $(\mathrm{p}=0.003)$, proteinuria $(\mathrm{p}=0.006)$ and arthritis $(\mathrm{p}=0.006)($ Table 1$)$. 
Table (1): Correlation between hyponatremia and different parameter of SLE activity, lab parameters and medication.

\begin{tabular}{|c|c|c|c|c|}
\hline \multirow[t]{2}{*}{ 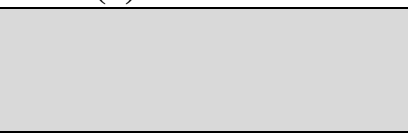 } & \multirow[t]{2}{*}{ Parameter } & \multicolumn{2}{|c|}{ SLE patients $(n=100)$} & \multirow[t]{2}{*}{$\mathbf{p}$} \\
\hline & & $\begin{array}{c}\text { Hyponatremia } \\
(n=41)\end{array}$ & $\begin{array}{l}\text { without hyponatremia } \\
(n=59)\end{array}$ & \\
\hline Age $\quad$ (years) & & $33.2 \pm 9.67$ & $34.84 \pm 12,3$ & 0.645 \\
\hline Gender F:M & & $36: 5$ & $56: 3$ & 0.032 \\
\hline Disease duration (months) & & $41.3 \pm 9.6$ & $48.2 \pm 11.7$ & 0.87 \\
\hline \multirow{9}{*}{ Clinical } & Vasculitis & 10 & 2 & 0.003 \\
\hline & Arthritis & 18 & 11 & 0.006 \\
\hline & malar Rash & 13 & 16 & 0.6 \\
\hline & New ulcer & 15 & 6 & 0.002 \\
\hline & Alopecia & 8 & 9 & 0.5 \\
\hline & Oral ulcer & 30 & 31 & 0.03 \\
\hline & Fever & 17 & 15 & 0.09 \\
\hline & New rash & 4 & 7 & 0.6 \\
\hline & Frothy urine & 23 & 13 & 0.001 \\
\hline \multirow[t]{7}{*}{ Lab } & Proteinuria & 22 & 12 & 0.006 \\
\hline & Pyuria & 5 & 7 & 0.002 \\
\hline & ALT (IU/L) & $20.8 \pm 4.08$ & $18.76 \pm 3.18$ & 0.322 \\
\hline & AST (IU/L) & $24.3 \pm 6.5$ & $22.4 \pm 4.05$ & 0.29 \\
\hline & Leucopenia & 11 & 4 & 0.09 \\
\hline & Thrombocytopenia & 10 & 4 & 0.5 \\
\hline & ESR (mm/hr.) & $65.23 \pm 13.94$ & $67.12 \pm 15.91$ & 0.7 \\
\hline \multirow[t]{4}{*}{ Medication } & Azathioprien & 23 & 27 & 0.4 \\
\hline & Mycnolate mofetil & 4 & 5 & 0.8 \\
\hline & Hydroquine & 15 & 35 & 0.04 \\
\hline & leflonamide & 3 & 7 & 0.5 \\
\hline SLEDAI & & $7.72 \pm 1.41$ & $3.9 \pm 0.913$ & $<0.0001$ \\
\hline
\end{tabular}

The Patients also were assessed by SLEDAI activity score and correlated with phosphorus level the patient with hypophosphatemia $(n=47)$ were 4 males and 43 females with a mean age of $32.8 \pm 9.75$ years while patients without ( $=53$ ) were 2 males and 51 females with a mean age of 35.66 \pm 12 , 40 years. The clinical manifestations in patients with and without hypophosphatemia show insignificant correlation with ESR $(p=0.5)$, low platelet $(p=0.9)$, low WBCs $(\mathrm{p}=0.1)$ and significant correlation with SELDIA score $(\mathrm{p}=<0.0005)$, proteinuria $(\mathrm{p}=0.002)$ and arthritis $(\mathrm{p}<0.0004)$ (Table 2).

Table (2): Correlation between hypophosphatemia and SLE activity parameter, lab findings and medications.

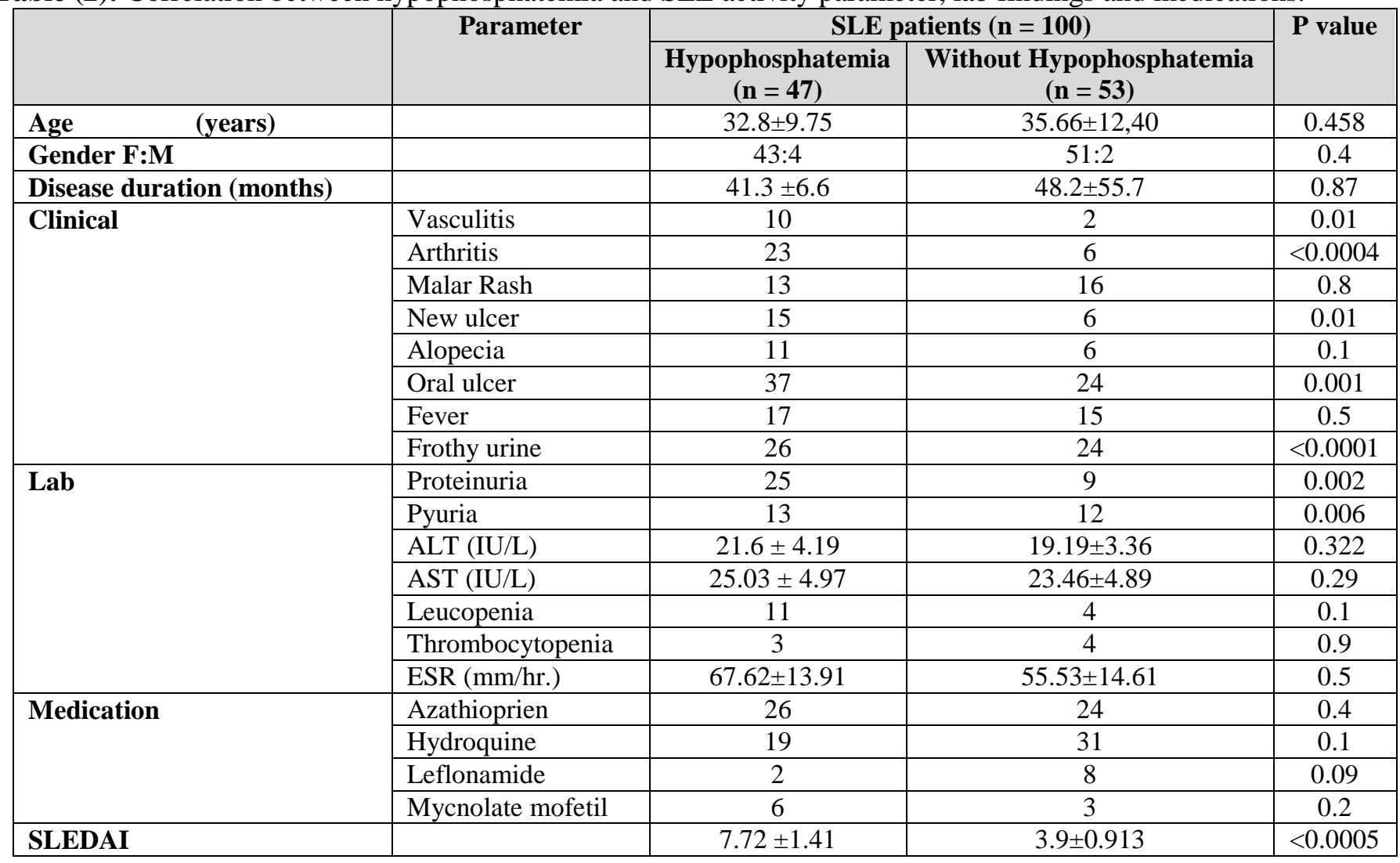


The patients were divided into no activity, mild, moderate and high disease and very high activity by SLEDIA, 17patient had no activity (SLEDAI=0) 28 patient had mild(SLEDAI $=1-5$ ), 25 had moderate disease activity $($ SLEDAI $=6-10), 24$ patient had severe disease activity (SLEDAI $=11-19)$ and 6 patient had very high disease activity $($ SLEDAI $=>20)$ in table 3 and compare them in hyponatremia and hypophosphatemia in figure 1 and figure 2 show that more hyponatremia and hypophosphatemia associated with more sever disease activity.

Table (3): Grading of severity of activity.

GRADING of severity of activity

\begin{tabular}{|c|c|c|c|}
\hline & & Frequency & Percent \\
\hline \multirow[t]{6}{*}{ Valid } & No & 17 & 17.0 \\
\hline & mild & 28 & 28.0 \\
\hline & moderate & 25 & 25.0 \\
\hline & severe & 24 & 24.0 \\
\hline & very high & & 6.0 \\
\hline & Total & 100 & 100.0 \\
\hline
\end{tabular}

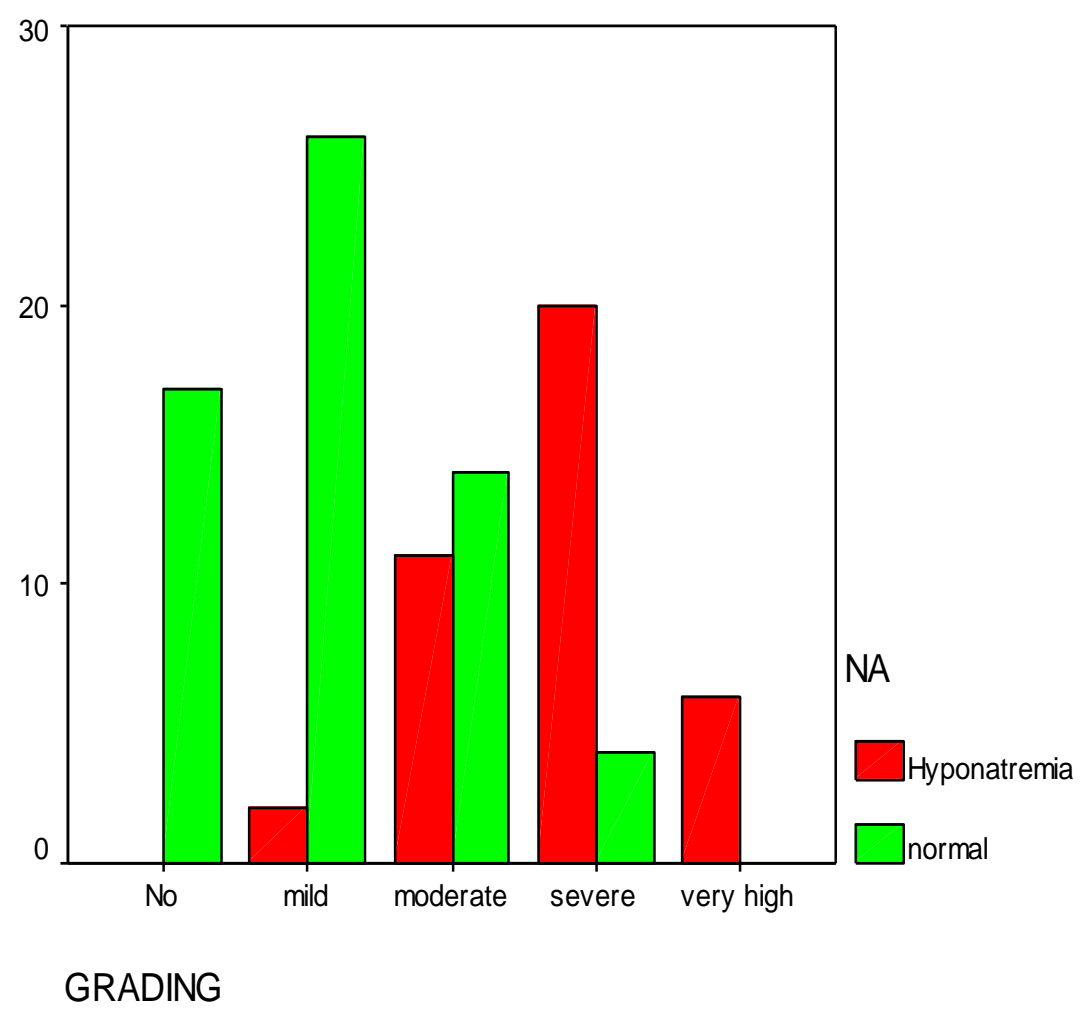

Figure (1): The relation between hyponatremia and grading of severity of activity. 


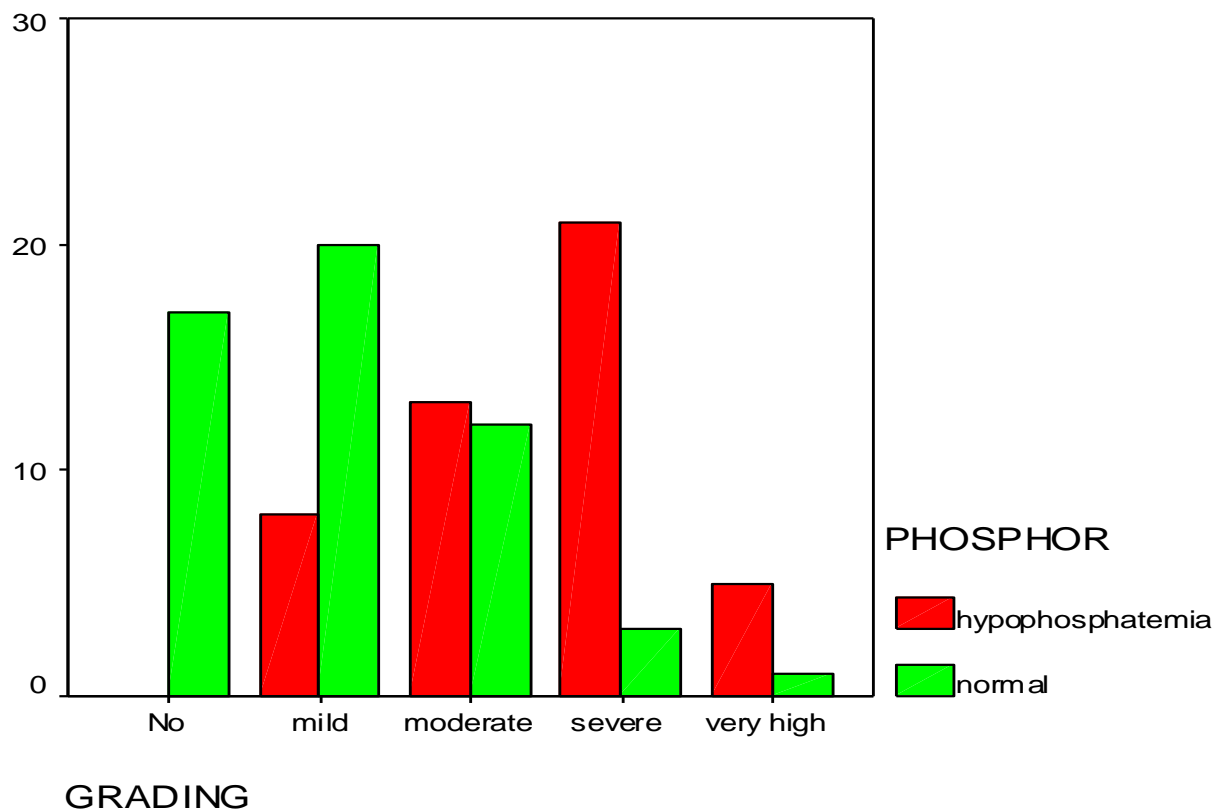

Figure (2): The relation between hypophosphatemia and grading of severity of activity.

\section{DISCUSSION}

Systemic lupus erythematosus (SLE) is an autoimmune disease in which the body's immune system mistakenly attacks healthy tissue in many parts of the body. Symptoms vary between people and may be mild to severe. Often there are periods of illness, called flares, and periods of remission during which there are few symptoms ${ }^{(21,22)}$. In our study 100 SLE patients were assessed by SLIDE activity score and correlated with sodium and phosphorus levels to investigate relation and effect of hyponatremia and hypophosphatemia as indicators for activity.

patients included in this study were 94 females (94\%) and 6 males $(6 \%)$ with a mean age of $34.23 \pm 11.19$ years. The age is symmetrical in distribution of age group to SLE affection and activity with agreement with other authors, El-Badawy et al. ${ }^{(16)}$ and Yamany ${ }^{(23)}$ and asymmetrical in distribution to Shin et al. ${ }^{(15)}$, who done his study on child and adult with SLE, and Fujiwara et al. ${ }^{(10)}$ who done his study on juvenile SLE patient.

In our study sex distribution wear 94 females (94\%) and 6 males (6\%), which is same as gender affection in other series Shin et al. ${ }^{(15)}$, El-Badawy et al. (16) and Yamany (23), with different gender distribution with other series e.g., Fujiwara et al. ${ }^{(10)}$ who did his study on female gender only and juvenile which may explain some differences in result.

The mean duration of the disease in our series was 48 months, with $\mathrm{SD} \pm 55.75$, and it ranged from 1 to 266 months. Same duration or near data from other series in comparison to our study $(\mathbf{1 5 , 1 6 , 2 3 )}$.

In the current study, patient activity was divided according to SLEDAI to active and inactive. Activity again subdivided into mild, moderate, high and very high. Using of this universal score put our patient series in head-to-head comparison with other authors ${ }^{(15}$, 16, 23).

The current work hypothesized that low serum sodium level and low phosphorus level could be a determinant of lupus inflammatory activity. Hence, the aim of the study design was to evaluate both hypophosphatemia and hyponatremia in lupus disease. The other variable factors including medications, namely diuretics, and renal sodium loss due to renal tubular injury and tubular membrane damage, leading to acute kidney injury (AKI) ${ }^{(24)}$. So, those patients with tubule-interstitial nephritis proven by renal biopsy were excluded from this study ${ }^{(23)}$.

In our study SLE patients were assessed by SLEDAI activity score, ESR WBCS, Platelets were correlated with sodium and phosphorus levels to investigate the relation and effect of hyponatremia and hypophosphatemia as indicators for activity.

We found that serum $\mathrm{Na}$ levels significantly correlated to vasculitis, arthritis and oral ulcers, all in favor that hyponatremia in SLE patients is directly related to more severe activity and inflammation. In accordance, the association of hyponatremia with SLEDAI, decreased C3 and increased ESR levels in juvenile SLE cases has been demonstrated in addition to a negative relation of serum sodium with serum IL-6 levels was found in adult SLE patients ${ }^{(\mathbf{1 5})}$.

Fujiwara et al. ${ }^{(10)}$ reported that the reason for significant correlation of serum $\mathrm{P}$ with anti-singlestranded DNA antibody, not with dsDNAab, is uncertain; however, it might be due to heterogeneity in pathogenesis of SLE.

In our study we found that hypophosphatemia is correlated positively to SLEDAI and arthritis and correlated negatively to ESR, platelet and WBCs. 
Further studies are necessary to study the pathophysiology of hypophosphatemia and hyponatremia in SLE.

\section{CONCLUSION}

It could be concluded that hyponatremia and hypophosphatemia are significantly related to SLEDAI, so it could be used as indicators of SLE activity and sever inflammation.

\section{Financial support and sponsorship: Nil.}

Conflict of interest: Nil.

\section{REFERENCES}

1. Maddison P (2002): Is it SLE? Best Pract Res Clin Rheumatol., 16:167-80.

2. Tamer G, Abdel Noor R, Abualfadl E et al. (2021): Adult systemic lupus erythematosus in Egypt: The nation-wide spectrum of 3661 patients and world-wide standpoint. Lupus, 2021: 09612033211014253.

3. Rahman A, Isenberg D (2008): Systemic lupus erythematosus. N Engl J Med., 358(9):929-39.

4. Riemekasten G, Hahn B (2005): Key autoantigens in SLE. Rheumatology (Oxford), 44(8):975-82.

5. Fischer-Betz R, Herzer P, Schneider M (2005): Systemic lupus erythematosus. Dtsch Med Wochenschr., 130(43): 2451-2458.

6. Kotzin B, O'Dell J (1995): Systemic lupus erythematosus. In: Frank MM, Austen KF, Claman HN, Unanue ER (eds). Samter's Immunologic Diseases. 5th edn. Lippincott Raven, Philadelphia, Pp. 667-97.

7. Arkachaisri T, Lehman T (1999): Systemic lupus erythematosus and related disorders of childhood. Curr. Opin. Reumatol., 11: 384-92.

8. Daifallah M, Azmy G, Abu Elfadl E (2020): Clinical significance of red blood cell distribution width in systemic lupus erythematosus patients. Egyptian Rheumatology and Rehabilitation, 47: 1-8.

9. Drezner M (2000): PHEX Gene And Hypophosphatemia. Kidney Kidney Int., 57: 9-18.

10. Fujiwara I, Ogawa E, Kondo Y et al. (2003): Hypophosphatemia in juvenile patients with systemic lupus erythematosus. Pediatrics International, 45(1):2330.

11. White K, Jonsson K, Carn Get al. (2001): The autosomal dominant hypophosphatemic rickets (ADHR) gene is a secreted polypeptide overexpressed by tumors that cause phosphate wasting. J Clin Endocrinol Metab., 86: 497-500.
12. Barak V, Schwartz A, Kalickman I et al. (1998): Prevalence of Hypophosphatemia in Sepsis and Infection: The Role of Cytokines. Am J Med., 104: 4047.

13. Adrogué $\mathbf{H}$, Madias N (2012): The Challenge of Hyponatremia. J Am Soc Nephrol., 23:1140-8.

14. Ayus J, Achinger S, Arieff A (2008): Brain Cell Volume Regulation in Hyponatremia: Role of Sex, Age, Vasopressin, And Hypoxia. Am J Physiol Renal Physiol., 295: 619-24.

15. Shin J, Park S, Suh C et al. (2016): Hyponatremia in patients with systemic lupus erythematosus. Sci Rep., 6:1-7.

16. El-Badawy M, El-Mahdi A, El-Sherbiny D et al. (2019): Hyponatremia in systemic lupus erythematosus patients: relation to disease activity and fatigue. The Egyptian Rheumatologist, 41(4): 283-287.

17. Martín J, Terroba M, Dib B et al. (1996): Systemic Lupus Erythematosus and the Syndrome of Inappropriate Secretion of Antidiuretic Hormone. Clin Exp Rheumatol., 14:578-9.

18. Elisaf M, Milionis H, Drosos A (1999): Hyponatremia Due to Inappropriate Secretion of Antidiuretic Hormone in A Patient with Systemic Lupus Erythematosus. Clin Exp Rheumatol., 17:223-6.

19. Petri M, Orbai A, Alarcón G et al. (2012): Derivation and validation of the Systemic Lupus International Collaborating Clinics classification criteria for systemic lupus erythematosus. Arthritis Rheum., 64(8):2677-86.

20. Bombardier C, Gladman D, Urowitz M et al. (1992): Derivation of the SLEDAI. A disease activity index for lupus patients. Arthritis Rheum., 35:630-40.

21. Lisnevskaia L, Murphy G, Isenberg D (2014): Systemic lupus erythematosus. Lancet, 384 (9957): 1878-88.

22. Gladman D (2017): Overview of the clinical manifestations of systemic lupus erythematosus in adults. UpToDate. https://www.uptodate.com/contents/overview-of-theclinical-manifestations-of-systemic-lupuserythematosus-in-adults

23. Yamany A, Behiry $M$, Ahmed $S$ (2020): Hyponatremia as an Inflammatory Marker of Lupus Activity Is a Fact or Fad: A Cross-Sectional Study. Open Access Rheumatology: Research and Reviews, 12 29-34.

24. Kinloch A, Chang A, Ko K et al. (2014): Vimentin is a dominant target of in situ humoral immunity in human lupus tubulointerstitial nephritis. Arthritis Rheum., 66(12): 3359-3370. 